\title{
A turnover pathway for both stable and unstable mRNAs in yeast: evidence for a requirement for deadenylation
}

\author{
Carolyn J. Decker and Roy Parker \\ Department of Molecular and Cellular Biology, University of Arizona, Tucson, Arizona 85721 USA
}

\begin{abstract}
To determine pathways of mRNA turnover in yeast, we have followed the poly(A) tail removal and degradation of a pulse of newly synthesized transcripts from four different genes. Before decay of both stable and unstable mRNAs initiated, there was a temporal lag during which the poly(A) tail was deadenylated to an oligo(A) length. Altering the deadenylation rate of an mRNA led to a corresponding change in the length of this lag. The rate of deadenylation and the stability of the oligo(A) species varied between mRNAs, explaining the differences in mRNA half-lives. To examine how the transcript body was degraded following deadenylation, we used the strategy of inserting strong RNA secondary structures, which can slow exonucleolytic digestion and thereby trap decay intermediates, into the 3' UTR of mRNAs. Fragments lacking the $5^{\prime}$ portion of two different mRNAs accumulated after deadenylation as full-length mRNA levels decreased. Therefore, these results define an mRNA decay pathway in which deadenylation leads to either internal cleavage or decapping followed by $5^{\prime} \rightarrow 3^{\prime}$ exonucleolytic degradation of the mRNA.
\end{abstract}

[Key Words: deadenylation; poly(A) tail; mRNA decay]

Received April 12, 1993; revised version accepted June 7, 1993.

Differences between the decay rate of mRNAs, as well as changes in the rate of decay of individual messages, can significantly affect the level of gene expression in eukaryotes. Although some differences in mRNA decay rates can be attributed to specific sequences within individual mRNAs, the role of such sequences and the mechanisms by which mRNAs are degraded are poorly defined (for review, see Cleveland and Yen 1989; Peltz et al. 1991).

The role of the poly(A) tail in mRNA decay also remains unresolved. Poly(A) tails are added to the $3^{\prime}$ end of mRNAs in the nucleus (for review, see Wickens 1990) and have been proposed to stabilize transcripts in the cytoplasm (Bernstein and Ross 1989). Because poly(A) tails generally undergo progressive deadenylation in the cytoplasm (Sheiness and Darnell 1973), it has been suggested that this removal of the poly(A) tail might lead to the degradation of mRNA. There is good evidence that rapid deadenylation is the first step in the decay of the unstable mammalian c-myc and c-fos mRNAs (Brewer and Ross 1988; Wilson and Triesman 1988; Swartwout and Kinniburgh 1989; Laird-Offringa et al. 1990; Shyu et al. 1991|. Whether deadenylation plays a general role in the degradation of mRNAs, however, is debatable because species of mRNAs with short or no poly(A) tail can be quite stable (Krowczynska et al. 1985; Herrick 1990; Shyu et al. 1991). Moreover, there are examples of unstable mRNAs being degraded by internal endonuclease cleavage independent of deadenylation (Binder et al.
1989; Stoeckle and Hanafusa 1989|. Thus, it is unclear whether degradation stimulated by deadenylation is a minor decay pathway, unique to a subset of unstable mRNAs, or whether it is a commonly used mechanism of mRNA decay.

Our recent analysis of mutations within the unstable yeast MFA2 mRNA suggested that, like in mammals, deadenylation is required for the decay of at least one yeast mRNA (Muhlrad and Parker 1992). We therefore wished to determine whether $\operatorname{poly}(\mathrm{A})$ tail removal is a general requirement for mRNA decay in yeast. To this end we developed a procedure that can be used to examine the degradation pathway of virtually any mRNA. This strategy utilizes an inducible promoter to produce a population of transcripts within a short period of time. Because these newly synthesized transcripts have poly(A) tails of discrete length, their size can be monitored with time to determine the relationship between poly(A) tail removal and degradation of the body of the transcript. In addition, this procedure allows us to determine unambiguously whether any mRNA fragments show a precursor-product relationship with the intact mRNA and are therefore products of transcript degradation. Our results using this procedure both define an mRNA decay pathway common to several mRNAs, in which deadenylation is the first step, and have important implications for understanding the basis of differential decay. 


\section{Results}

\section{Strategy}

The approach that we used to examine mRNA degradation pathways was to place the gene encoding the transcript of interest under the regulation of the GAL1 upstream activating sequence (UAS). This gene was then introduced into yeast cells carrying a temperature-sensitive mutation in RNA polymerase II, $r p b 1-1$ (Nonet et al. 1987). The cells were pregrown at $24^{\circ} \mathrm{C}$ on raffinose, which maintains the GAL1 UAS in an inactive state (Johnston 1987), and transcription was induced rapidly by the addition of galactose. After a brief period of time (10-15 $\mathrm{min}$ ), transcription was rapidly repressed by shifting the strain to $36^{\circ} \mathrm{C}$, inactivating the thermolabile polymerase, and adding glucose, a catabolite repressor of the GAL1 UAS. Both means of transcriptional repression were used because this resulted in tighter and faster inhibition than the use of either means alone /data not shown). In addition, similar results were obtained when either means of repression was used alone, indicating that the temperature shift or the change in carbon source did not significantly affect deadenylation or mRNA decay rates (see Materials and methods). At least $85 \%$ of the mRNA present when transcription was repressed was produced during the pulse, with the majority of the transcripts being produced in the last few minutes (data not shown). Aliquots were then harvested at various times, and the rate of transcript deadenylation and the decay rate of the transcript body were examined. Deadenylation rates were calculated by running total RNA from each time course on acrylamide Northern gels and measuring the length of the shortest poly(A) tails at each time point by comparison with a sample where the poly(A) tail had been removed by treatment with oligo(dT) and RNase H. For longer mRNAs, this analysis required that the body of the transcript be shortened by cleavage with an oligonucleotide complementary to sequences near its $3^{\prime}$ end and RNase H. Decay of the
mRNA body was measured by comparing the amount of mRNA remaining at each time point relative to the amount present at the time of transcriptional repression normalized to an internal control (see Materials and methods).

We used this approach to examine the decay of four yeast mRNAs of varying stability. Two transcripts examined, those arising from the $M F A 2\left(t_{1 / 2}=3.5 \mathrm{~min}\right)$ and $S T E 3\left(t_{1 / 2}=3.8 \mathrm{~min}\right)$ genes are highly unstable, whereas the PGK1 $\left(t_{1 / 2}=45 \mathrm{~min}\right)$ and $G A L 10\left(t_{1 / 2}=25 \mathrm{~min}\right)$ transcripts are relatively stable (Herrick et al. 1990 and data not shown). For each of these mRNAs we observed the same general metabolism of the poly/A) tail (see below). The transcripts produced during the transcriptional burst had long poly(A) tails of relatively discrete length (see Table 1), although some length variation was observed because the population of transcripts will contain mRNAs of slightly different ages [suggested by the observation that at very early times of induction the poly(A) tail size was more homogeneous (data not shown)]. These long poly(A) tails shortened with time to produce transcripts with an average of 7-16 adenylate residues remaining. We refer to these mRNAs as having an oligo(A) tail.

\section{The decay of several yeast $m R N A$ s requires $a$ time-dependent event}

Beginning with the stable PGK1 mRNA we compared the time when decay initiated to the time it took for deadenylation to occur. If deadenylation is a prerequisite for the decay of an mRNA, degradation of the transcript body should not begin until after deadenylation. Decay of the pool of transcripts, however, would be expected to initiate immediately if deadenylation is not required.

Decay of the PGK1 mRNA did not begin until $\sim 30$ min after transcription was repressed (Fig. 1). During the time interval between 0 and $30 \mathrm{~min}$, the poly(A) tails of the PGK1 transcripts shortened from an initial length of

Table 1. Poly $(A)$ tail length and deadenylation rates for stable and unstable mRNAs

\begin{tabular}{|c|c|c|c|c|}
\hline \multirow[b]{2}{*}{ mRNA } & \multicolumn{2}{|c|}{ Length of poly(A) tail (range in no. of A residues) } & \multicolumn{2}{|c|}{ Rate of deadenylation (A residues per min) } \\
\hline & initial $^{\mathrm{a}}$ & oligo $(A)^{b}$ & initial deadenylation $^{c}$ & poly $|A|$ shortening ${ }^{d}$ \\
\hline PGK1 & $55 \pm 14$ to $72 \pm 17$ & $5 \pm 2$ to $13 \pm 1$ & $1 \pm 0.5$ & $4 \pm 2$ \\
\hline GAL10 & $45 \pm 5$ to $58 \pm 6$ & $3 \pm 1$ to $13 \pm 1$ & $1.5 \pm 0.5$ & $8 \pm 1$ \\
\hline MFA2 & $55 \pm 10$ to $88 \pm 12$ & $11 \pm 3 \quad$ N.D. ${ }^{\mathrm{e}}$ & $2 \pm 0.5$ & $13 \pm 2$ \\
\hline STE3 & $39 \pm 3$ to $62 \pm 10$ & $10 \pm 2$ to $21 \pm 6$ & $4 \pm 2$ & $20 \pm 6$ \\
\hline
\end{tabular}

${ }^{a}$ Range in number of adenylate residues present on the induced pool of transcripts at the time when transcription was first repressed, determined as described in Results. Each figure is the mean of multiple experiments with the standard error as shown.

${ }^{b}$ Range in number of adenylate residues present on the shortest observed poly $(A)$ tail species.

${ }^{c}$ Change in the length of the shortest poly(A) tail species with time expressed as the number of adenylate residues per minute. Rate of deadenylation observed in the time points just after transcriptional repression. The rates measured for the STE3 and MFA2 mRNAs are likely to be overestimates due to the population of transcripts containing mRNAs that may have already entered the rapid poly(A) shortening phase when transcription was repressed.

${ }^{\mathrm{d}}$ Rate of deadenylation observed after initial deadenylation.

eAn upper range of the oligo(A) species of MFA2 mRNA could not be measured because oligoadenylated species did not accumulate to a great enough extent above its heterogeneous distribution of poly(A) tail lengths (see Fig. $3 \mathrm{~A}$ ). 
A

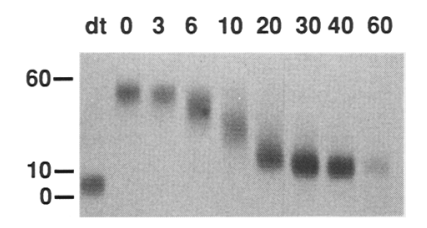

minutes after inhibition of transcription

B

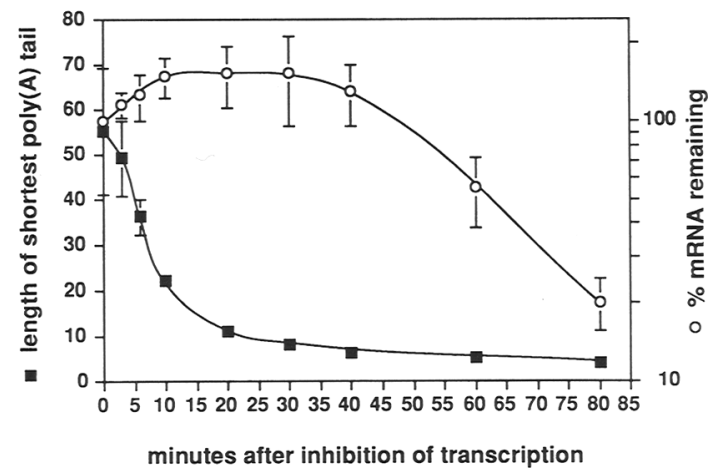

Figure 1. PGK1 mRNA decay and deadenylation. (A) Polyacrylamide Northern blot of a pool of PGK1 transcripts produced during a 15-min induction with galactose. Samples were taken at various times after transcriptional repression as indicated. Total RNA from each sample was treated with RNase $\mathrm{H}$ in the presence of the oligonucleotide RP70, CGGATAAGAAAGCAACACCTGG, which is complementary to the sequence 9-30 nucleotides $5^{\prime}$ of the PGK1 stop codon. The first lane is RNA treated with RNase $\mathrm{H}$, the $P G K 1$-specific oligonucleotide, and oligo(dT) to remove the poly $(\mathrm{A})$ tail. The distribution in size of the mRNA fragments in each sample is attributable to poly(A) tail length differences because these size differences are no longer seen when the samples are treated with oligo(dT) and RNase $\mathrm{H}$ (first lane in each figure; data not shown). The probe was an end-labeled oligonucleotide complementary to a 42-nucleotide sequence inserted $3^{\prime}$ of the stop codon. This insertion has no effect on PGK1 mRNA stability (Heaton et al. 1992; Parker et al. 1992). The numbers at left represent the approximate migration of the fragment with the indicated number of adenylate residues. $(B)$ The decay and deadenylation rates of the pool of $P G K 1$ transcripts. The length of the shortest poly(A) tail at the various time points and the decay rate were calculated as described in Results. The points represent the mean of three experiments, two in yRP583 and one in yRP582. The increase in RNA levels seen during the early time points is most likely attributable to incomplete repression of transcription.

$\sim 55-70$ adenylate residues to an oligo(A) tail of $\sim 5-13$ residues, with a maximum rate of $\sim 4$ adenylate residues per minute. Thus, the amount of $P G K 1$ mRNA did not begin to decrease until the transcripts were deadenylated to an oligo(A) form, suggesting that the decay of PGK1 mRNA required prior shortening of its poly $(\mathrm{A})$ tail (see Fig. 1B).

There was also a delay in the decay of the two unstable mRNAs examined, MFA2 and STE3 (Fig. 2 and 3). Decay of MFA2 mRNA did not initiate until between 4 and 6 min after transcriptional repression. Unlike the PGK1 transcripts, the $M F A 2$ transcripts were not uniformly deadenylated; however, by $4 \mathrm{~min}$ a significant fraction of the pool had an oligo(A) tail (Fig. 2). The rate of poly(A) shortening of $M F A 2$ transcripts, $\sim 13$ adenylate residues per minute, was significantly faster than that seen on PGK1 transcripts. There was a similar lag, although quite brief, before STE3 mRNA achieved its maximum rate of decay (Fig. 3B). Again this interval corresponded to the time it took for a large fraction of the STE3 transcripts to be deadenylated to an oligo(A) form (Fig. 3). The fact that the STE3 transcripts decayed to some ex-

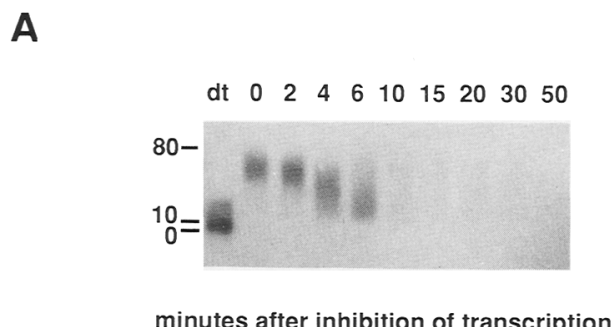

B

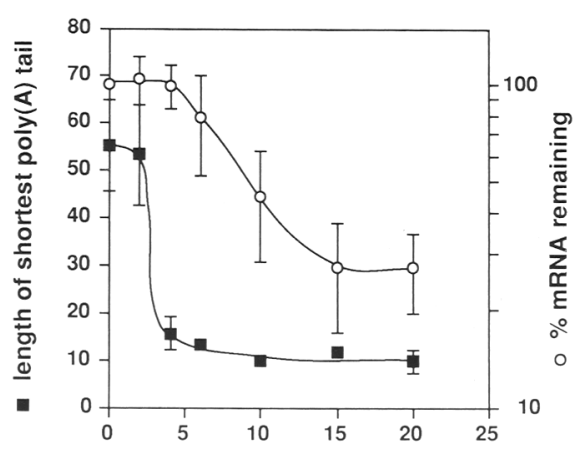

minutes after inhibition of transcription

Figure 2. MFA2 mRNA decay and deadenylation. $(A \mid$ Polyacrylamide Northern blot of a pool of MFA2 transcripts produced during a 10-min induction with galactose. Samples were taken at various times after transcriptional repression as indicated. The first lane is RNA treated with RNase $\mathrm{H}$ and oligo(dT) to remove the poly(A) tail. The two bands seen in this lane represent species with different $3^{\prime}$ ends (Muhlrad and Parker 1992). The probe was a random prime-labeled SphI-NdeI fragment containing the $M F A 2$ gene. The numbers at left represent the approximate migration of the mRNA with the indicated number of adenylate residues. $(B)$ The decay and deadenylation rates of the pool of MFA2 transcripts. The length of the shortest poly(A) tail at the various time points and the decay rate were calculated as described in Results. The points represent the mean of four experiments, three in yRP583 and one in yRP673. Approximately $30 \%$ of the MFA2 mRNA does not decay over the time course and maintains a heterogeneous poly(A) tail size distribution. We do not know the reason for this phenomenon, although it is unlikely to be attributable to MFA2 mRNA decay requiring a labile factor because this result was also seen when the temperature shift was omitted; however, it might be attributable to incomplete inhibition of transcription. 
A

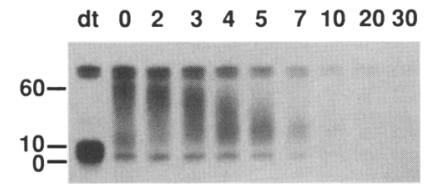

minutes after inhibition of transcription

B

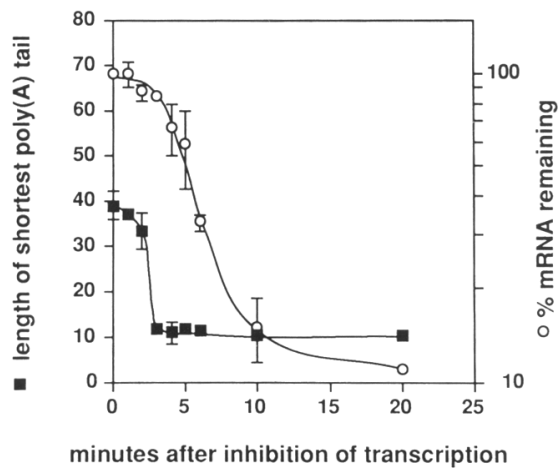

Figure 3. STE3 mRNA decay and deadenylation. $(A)$ Polyacrylamide Northern blot of a pool of STE3 transcripts produced during a 10-min induction with galactose. Samples were taken at various times after transcriptional repression as indicated. Total RNA from each sample was treated with RNase $\mathrm{H}$ in the presence of the oligonucleotide RP15, AATGCGACACTCTTGTGTTA, which is complementary to the translational stop codon and 17 nucleotides downstream of the stop codon. The first lane is RNA treated with RNase $\mathrm{H}$, the STE3-specific oligonucleotide, and oligo(dT) to remove the poly(A) tail. The probe was an in vitro-synthesized antisense transcript of a HindIII fragment containing the $3^{\prime}$ end of STE3 $\mathrm{mRNA}$ and that extends $5^{\prime}$ of the site of RP15. The somewhat diffuse pattern of STE 3 3' fragments at the early time points is likely to be attributable to the extremely rapid deadenylation of this transcript in relationship to the time of the transcriptional pulse. The bands seen just above and below the diffuse signal representing the STE3 3' fragment result from additional RNase $\mathrm{H}$ cleavages as a result of RP15 hybridizing to additional sites in the STE3 mRNA because they are not seen when another STE3-specific oligonuceotide is used in the RNase $\mathrm{H}$ reactions /data not shown). The numbers at left represent the approximate migration of the fragment with the indicated number of adenylate residues. $(B)$ The decay and deadenylation rates of the pool of STE3 transcripts. The length of the shortest poly(A) tail on the bulk of the new transcripts at the various time points and the decay rate were calculated as described in the results. The points represent the mean of four experiments, three in yRP673 and one in yRP582.

tent immediately following repression of transcription is likely to be attributable to the extremely rapid deadenylation of this transcript $\mid \sim 20$ adenylate residues per minute) in relationship to the time of the transcriptional pulse. Therefore, the time-dependent event required for the decay of MFA2 and STE3 mRNAs correlated with deadenylation to an oligo (A) form.
GAL10 transcripts also showed a delay in the onset of decay, but, unlike the other mRNAs, the length of this lag was longer than the time it took for a fraction of the GAL10 mRNA to be deadenylated (Fig. 4). Formally, these results could be explained by GAL10 mRNA being degraded by a mechanism that required a time-dependent event other than poly(A) shortening. Alternatively, deadenylation may be the only time-dependent event required for GAL10 mRNA decay, but the exact time when decay initiated was obscured because the population of transcripts was not deadenylated uniformly. For instance, at $10 \mathrm{~min}$, only $\sim 5 \%$ of the population was oligoadenylated (see legend to Fig. 4). Slow degradation of this small amount of RNA would not be detectable. In contrast, at $20 \mathrm{~min}, \sim 15 \%$ of the transcripts had oligo(A) tails, a percentage large enough to observe a decrease in mRNA levels between 20 and $40 \mathrm{~min}$. The observed decay rate would then be expected to accelerate because at 40 min at least $30 \%$ of the RNA was oligoadenylated.

\section{Deadenylation is a prerequisite for the decay of some yeast mRNAs}

The above experiments demonstrated that there was a delay of variable time for each of these mRNAs following inhibition of transcription before the initiation of degradation of the transcript. The length of this delay for at least three of the transcripts closely corresponded with the time it took for that transcript to deadenylate, suggesting that deadenylation is required for the decay of these mRNAs. These results, however, could also be explained by a different time-dependent event, independent of deadenylation, being required for decay that fortuitously corresponded to the deadenylation time for each mRNA. If deadenylation is required, then changing the rate at which the poly $(\mathrm{A})$ tail of an mRNA is shortened should lead to a change in the length of the delay in the onset of decay. We therefore examined the decay of an mRNA whose deadenylation rate was altered.

We constructed a chimeric mRNA in which we replaced the $3^{\prime}$-untranslated region (UTR) of the stable PGK1 mRNA with the $3^{\prime}$ UTR of the unstable MFA2 transcript (see Fig. 5A), which contains sequences that stimulate deadenylation (Muhlrad and Parker 1992). As shown in Figure 5B, this chimeric transcript deadenylated rapidly, reaching an oligo(A) form by 10 min rather than $30 \mathrm{~min}$ as seen for PGK1 mRNA (Fig. 1A). The chimeric transcript also began to decay at an earlier time than the parental PGK1 transcript (Fig. 5C). Because the chimeric PGK1-MFA2 transcript had an altered deadenylation rate, and a corresponding shift in the time at which decay initiated, we conclude that deadenylation is a prerequisite for the degradation of the body of PGK1 mRNA.

For PGK1 and MFA2 transcripts deadenylation does not lead to $3^{\prime}$ exonucleolytic degradation of the mRNA body

Once deadenylated, how is the transcript body degraded? One possibility is suggested by the work of Vreken and 
A

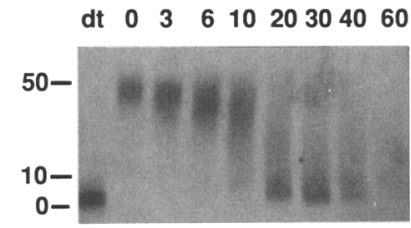

minutes after inhibition of transcription

B

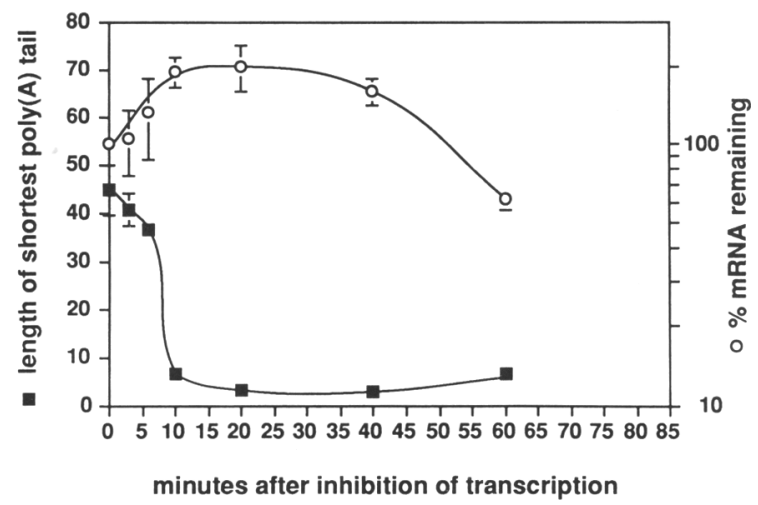

Figure 4. GAL1O mRNA decay and deadenylation. $(A)$ Polyacrylamide Northern blot of a pool of GAL10 transcripts produced during a $15-\mathrm{min}$ induction with galactose. Samples were taken at various times after transcriptional repression as indicated. Because the sequence of the entire GAL10 gene has not been determined in Saccharomyces cerevisiae, an oligonucleotide complementary to the sequence of GAL10 in the closely related yeast Saccharomyces carlsbergensis was used to cleave GAL10 mRNA. Total RNA from each sample was treated with RNase $\mathrm{H}$ in the presence of the oligonucleotide RP97, whose sequence is GTATCTACAAGGCTCGATTG and is complementary to 90-110 nucleotides $5^{\prime}$ of the stop codon (Citron et al. 1984). The first lane is RNA treated with RNase $\mathrm{H}$, the GAL10specific oligonucleotide, and oligo(dT) to remove the poly $(\mathrm{A})$ tail. The probe was an end-labeled oligonucleotide complementary to 18-38 nucleotides $5^{\prime}$ of the stop codon of $S$. carlsbergensis GAL10 mRNA. The numbers at left represent the approximate migration of the fragment with the indicated number of adenylate residues. $(B)$ The decay and deadenylation rates of the pool of GAL1O transcripts. The length of the shortest poly(A) tail at the various time points and the decay rate were calculated as described in Results. The points represent the mean of three separate experiments, all in yRP583. The graph does not include the 30 -min time point seen in $A$ because this time point was taken only in that particular experiment. The increase in RNA levels seen during the early time points is most likely attributable to incomplete repression of transcription. The percentage of mRNA that was oligoadenylated at any given time was determined by quantitating, using a Betascope, the amount of hybridization in the lower portion of the distribution and comparing that with the amount of hybridization in the entire distribution.

Raué (1992), who used the general strategy of trapping extremely unstable decay intermediates by the introduction of strong RNA secondary structures, which can slow or block exonucleolytic digestion (e.g., McLaren et al. 1991). They observed that introduction of secondary structures into the $3^{\prime}$ UTR of the yeast PGK1 mRNA leads to the accumulation of a fragment missing the $5^{\prime}$ end but not the $3^{\prime}$ end of the mRNA. This $3^{\prime}$ fragment is seen when either poly $(G)$, which forms an extremely strong secondary structure (Zimmerman et al. 1975; Williamson et al. 1989), or a stem-loop made up of G and $\mathrm{C}$ residues is inserted. The accumulation of this $3^{\prime}$ fragment suggested that the degradation of the body of the $P G K 1$ transcript proceeds in a $5^{\prime} \rightarrow 3^{\prime}$ direction, although a precursor-product relationship between the

A

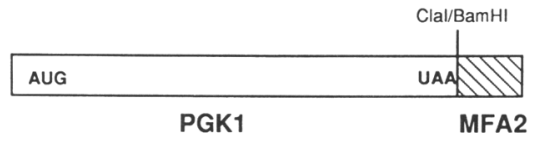

B

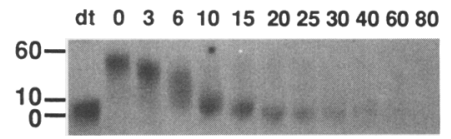

minutes after inhibition of transcription

C

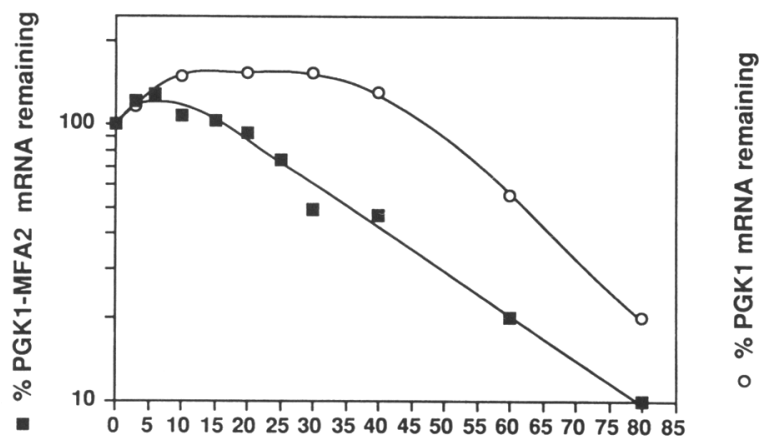

minutes after inhibition of transcription

Figure 5. Deadenylation and decay of chimeric PGK1-MFA2 transcripts. (A) Replacement of PGK1 3' UTR with that of MFA2 mRNA (see Materials and methods). The cartoon represents the resulting chimeric mRNA. The open box depicts the PGK1 sequence; the hatched box indicates the MFA2 sequence. (B) Polyacrylamide Northern blot of a pool of PGK1-MFA2 chimeric transcripts produced during a $15-\mathrm{min}$ induction. Samples were taken at various times after transcriptional repression as indicated. Total RNA from each sample was treated with RNase $\mathrm{H}$ in the presence of the PGK1-specific oligonucleotide as described in Fig. 1. The probe used was the same as that in Fig. 2. The numbers at left represent the approximate migration of the fragment with the indicated number of adenylate residues. $(C)$ Graph comparing the decay profile of PGK1-MFA2 chimeric mRNA to that of $P G K 1 \mathrm{mRNA}$. The points represent the mean of three separate experiments, two in yRP582 and one in yRP583. 
full-length mRNA and the fragment was not established. More importantly, because the analysis of this $3^{\prime}$ fragment was limited to steady-state conditions, it was not possible to determine whether deadenylation of the PGK1 mRNA was a requirement for the production of this $3^{\prime}$ fragment. Because our results indicated that deadenylation was a prerequisite for decay, these observations raised the possibility that deadenylation of the PGK1 mRNA leads to $5^{\prime} \rightarrow 3^{\prime}$ degradation of the transcript body. Alternatively, removal of the poly(A) tail has been proposed to stimulate degradation by exposing the $3^{\prime}$ end of mRNAs to $3^{\prime} \rightarrow 5^{\prime}$ exonuclease digestion (Brawerman 1987; Higgins 1991; Peltz et al. 1991; Shyu et al. 1991).

To determine the directionality of decay following deadenylation we inserted poly $(G)$ into the $3^{\prime}$ UTR of the PGK1 mRNA (see Materials and methods and Fig. 6A) and looked for the accumulation of any mRNA fragments that appeared after deadenylation. If deadenylation stimulates $3^{\prime} \rightarrow 5^{\prime}$ degradation of mRNAs, introduction of a strong secondary structure into the $3^{\prime}$ UTR of an mRNA might cause the accumulation of fragments that are missing the $3^{\prime}$ end of the mRNA. Alternatively, if deadenylation leads to $5^{\prime} \rightarrow 3^{\prime}$ degradation of an mRNA, we would expect a fragment corresponding to the $3^{\prime}$ portion of the transcript to appear after deadenylation.

By several criteria, including the overall decay profile, the deadenylation rate, and the half-life of the oligo(A) species, the insertion of the poly $(G)$ did not affect the decay of the PGK1 transcript (Fig. 6B,C). Thus, this slightly altered mRNA was degraded by the same mechanism as the parental PGK1 mRNA. An mRNA fragment of $\sim 170$ nucleotides was observed, which showed a precursor-product relationship with the full-length PGK1 transcripts and only began to appear after deadenylation (Fig. 6). This fragment extends from the poly $(G)$ insertion to the $3^{\prime}$ end of the PGK1 transcript based on the size of the fragment, its ability to hybridize to a poly $(\mathrm{C})$ probe, and the fact that it was oligoadenylated (as described below). By comparing the amounts of the fragment and the full-length mRNA present at various times, we calculate that at least $40 \%$ of the PGK1 mRNA molecules must be degraded by a pathway that leads to the production of this fragment. Because this estimate does not take into account any decay of the fragment, these data are consistent with the majority, if not all, of PGK1 mRNA being degraded in this manner. Because the fragment only appeared after the poly(A) tail had been shortened, we conclude that the role of deadenylation of the PGK1 transcript is to lead to the $5^{\prime} \rightarrow 3^{\prime}$ degradation of the mRNA, initiated either by endonucleolytic cleavage or removal of the $5^{\prime}$ cap structure.

It remains possible that unstable mRNAs, unlike PGK1 mRNA, may be degraded in a $3^{\prime} \rightarrow 5^{\prime}$ direction. This hypothesis is consistent with the observation that in mammals one unstable mRNA, c-myc, is degraded in vitro $3^{\prime} \rightarrow 5^{\prime}$ following deadenylation (Brewer and Ross 1988). To examine this possibility, we inserted a poly(G) tract into the $3^{\prime}$ UTR of the unstable MFA2 mRNA (Fig.
A

\begin{tabular}{|ll|l|}
\hline AUG & UAA & PG \\
\hline
\end{tabular}

B

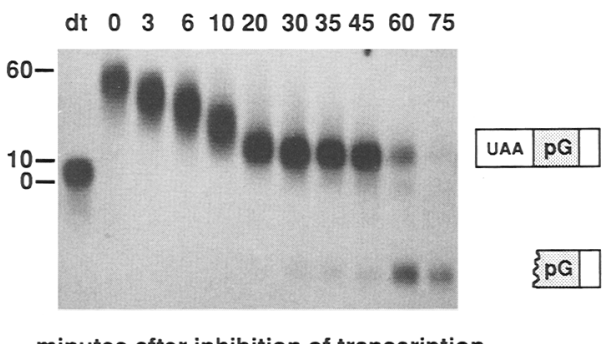

minutes after inhibition of transcription

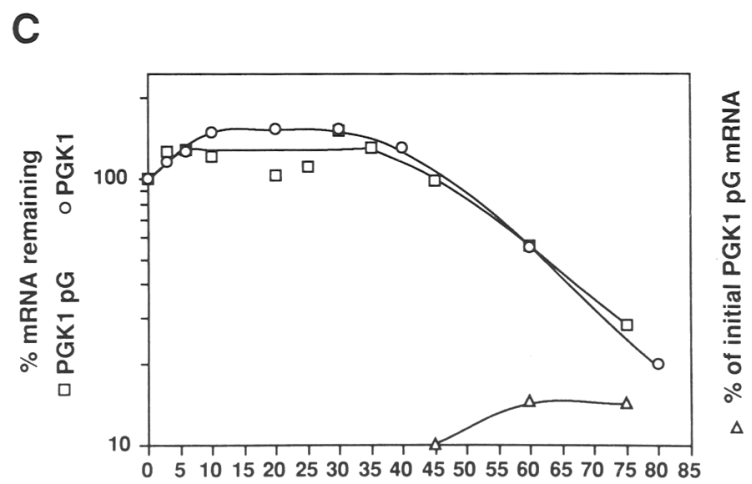

Figure 6. Degradation of PGK1 mRNA does not proceed $3^{\prime} \rightarrow 5^{\prime}$. (A) Insertion of a strong secondary structure to block exonuclease digestion into the 3' UTR of PGK1 mRNA. Eighteen guanylate residues were inserted into the ClaI site of $P G K 1$ just downstream of the translational stop codon as described in Materials and methods. The cartoon represents the resulting mRNA. The open box depicts the PGK1 sequence; the stippled box indicates the poly $(G)$ insertion. $(B)$ Polyacrylamide Northern blot of a pool of $P G K 1 p G$ transcripts produced within a 15-min induction. Samples were taken at various times after transcriptional repression as indicated. Total RNA from each sample was treated with RNase $\mathrm{H}$ in the presence of the PGK1specific oligonucleotide as described in Fig. 1. The probe used was an end-labeled oligonucleotide complementary to the poly $(G)$ insertion. The numbers at left represent the approximate migration of the fragment with the indicated number of adenylate residues. $(C)$ Comparison of the decay rate of PGK1 mRNA with and without the poly $(G)$ insertion. $(\triangle)$ The percentage of the initial PGK1pG mRNA that is represented by the $3^{\prime}$ fragment. The $P G K 1 p G$ mRNA points represent the mean of three separate experiments, all in yRP582.

7A). The poly $(G)$ insertion had no significant effect on the decay of the MFA2 transcript (Fig. 7C). A fragment of $\sim 190$ nucleotides began to accumulate substantially by 6 min after transcriptional inhibition (Fig. 7B,C), coinciding with the time that a large fraction of the pool of RNA was deadenylated to an oligo(A) form and the 
A

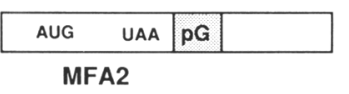

B

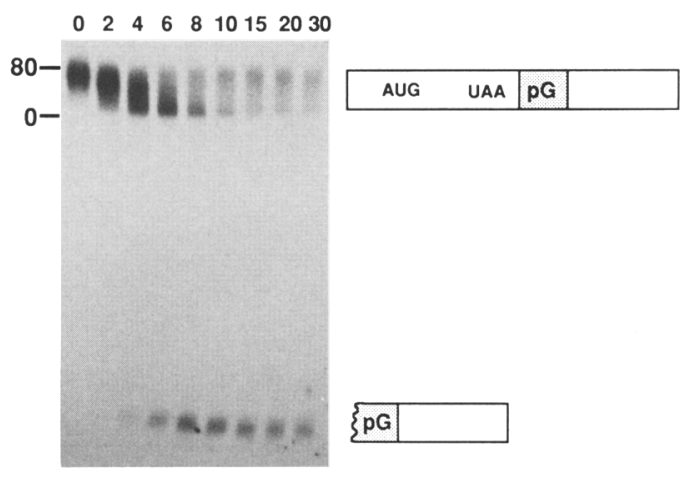

minutes after inhibition of transcription

C

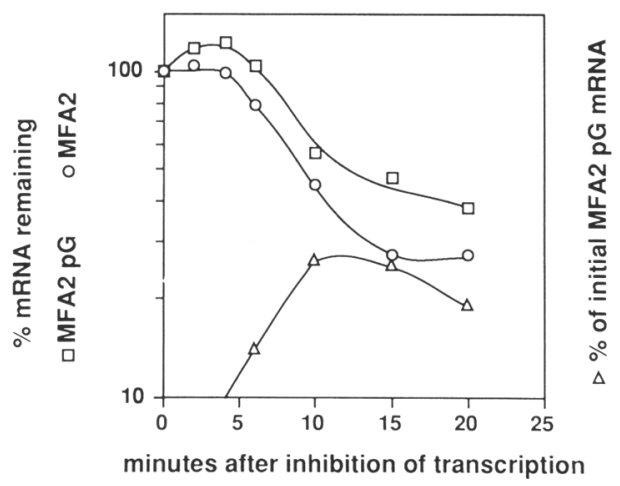

Figure 7. Degradation of MFA2 mRNA does not proceed $3^{\prime} \rightarrow 5^{\prime}$. $(A)$ Insertion of a strong secondary structure to block exonuclease digestion into the 3' UTR of MFA2 mRNA. Eighteen guanylate residues were inserted into a previously constructed BglII site (B178; Muhlrad and Parker 1992) just downstream of the MFA2 translational stop codon as described in Materials and methods. The cartoon represents the resulting mRNA. The open box indicates MFA2 sequence; the stippled box represents the poly $(G)$ insertion. $(B)$ Polyacrylamide Northern blot of a pool of $M F A 2 p G$ transcripts produced within a 10-min induction. Samples were taken at various times after transcriptional repression as indicated. The probe used was the same as that used in Fig. 6. The numbers at left represent the approximate migration of the mRNA with the indicated number of adenylate residues. $(C)$ Comparison of the decay rate of MFA2 mRNA with and without the poly $(G)$ insertion. $(\triangle)$ The percentage of the initial $M F A 2 p G$ mRNA that is represented by the 3 ' fragment. The $M F A 2 p G$ mRNA points represent the mean of three separate experiments, all in yRP582.

mRNA began to be degraded. The levels of the fragment require that at least $50 \%$, if not all, of the MFA2 mRNA was degraded through a pathway that generated this fragment. On the basis of its hybridization with different probes and RNase protection assays, this fragment corresponded to the $3^{\prime}$ end of the mRNA extending from the $5^{\prime}$ end of the poly $(G)$ tract to the $3^{\prime}$ end of the transcript (data not shown). Thus, like PGK1 mRNA, deadenylation of MFA2 mRNA led to the $5^{\prime} \rightarrow 3^{\prime}$ degradation of the mRNA.

In principle, the substrate for the degradation of the body of the PGK1 and MFA2 mRNAs could be molecules that have oligo(A) tails, or degradation could require that the transcript be completely deadenylated. To distinguish between these two possibilities, we examined whether the $3^{\prime}$ fragments had oligo(A) tails at the time they were first produced. To do this, we compared the size of the $3^{\prime}$ fragments at early time points in their accumulation with or without prior treatment with oligo(dT) and RNase $\mathrm{H}$ to remove any residual oligo(A) tail. As shown in Figure 8, the $3^{\prime}$ fragments from both the PGK1 and MFA2 transcripts had oligo(A) tails. Thus, it is not necessary for the mRNA to undergo complete removal of the poly(A) tail to be a substrate for the next step in degradation.

\section{Discussion}

Many yeast $m R N A$ s require deadenylation before decay initiates

To determine the pathways of mRNA decay in yeast, we examined the turnover of a synchronous pool of tran-

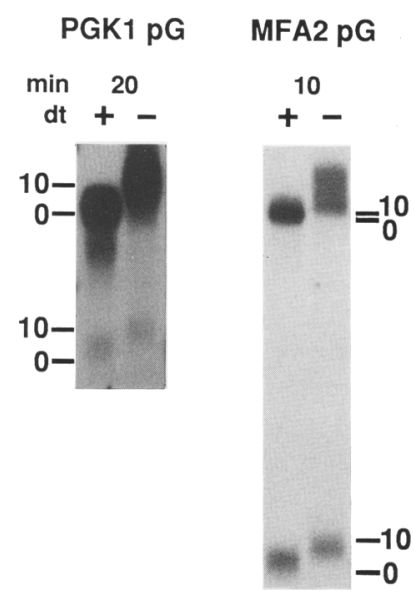

Figure 8. Degradation of $P G K 1$ and MFA2 mRNAs does not require complete removal of the poly(A) tail. Comparison of the size of the $3^{\prime}$ degradation intermediates of PGK1 and MFA2 mRNA with $(+\mid)$ and without $(-)$ treatment with RNase $\mathrm{H}$ and oligo(dT). Polyacrylamide Northern blots of PGK1pG and $M F A 2 p G$ mRNA samples were harvested at the time indicated after transcription was repressed. These time points represent early time points in the accumulation of the $3^{\prime}$ fragments. At later time points, the oligo(A) tails were shorter than those observed at these early time points (data not shown). The probe used was the same as that in Fig. 6. The PGK1pG samples were treated with RNase $\mathrm{H}$ and the $P G K 1$-specific oligonucleotide as described in Fig. 2. The upper band in each sample represents the full-length mRNA. The lower band represents the $3^{\prime}$ fragment. The numbers at the side represent the approximate migration of the mRNA and $3^{\prime}$ fragment with the indicated number of adenylate residues. 
scripts of both stable and unstable mRNAs. In these experiments there was a delay after transcriptional repression before mRNA decay commenced. Several observations suggest that this temporal delay represents a requirement for prior deadenylation of the mRNAs. First, for at least three of the mRNAs examined, the onset of decay closely correlated with the length of time it took for deadenylation to occur. Second, increasing the deadenylation rate of PGK1 mRNA led to a decrease in the length of the delay in its decay. Third, point mutations that increased the stability of MFA2 mRNA and slow deadenylation increased the length of the lag in the decay of MFA2 mRNA (Muhlrad and Parker 1992 and C. Beelman and R. Parker, unpubl.). Finally, decay intermediates began to appear only after a substantial fraction of the mRNA had been deadenylated. Moreover, these intermediates had oligo(A) tails, even at times when the mRNA population consisted of transcripts with both long and short poly(A) tails (Fig. 7), arguing that only oligoadenylated transcripts are substrates for the next step in decay. Our data therefore indicate that both stable and unstable mRNAs in yeast are degraded by a similar mechanism that requires prior deadenylation of the mRNA. It cannot be formally ruled out, however, that other time-dependent events may also be required for decay. In addition, it is likely that decay mechanisms that do not depend on prior deadenylation also exist because decay of PGK1 mRNA containing an early nonsense codon does not require deadenylation (D. Muhlrad and R. Parker, unpubl.) and there are examples, in other eukaryotes, of messages being cleaved endonucleolytically independent of deadenylation (Binder et al. 1989; Stoeckle and Hanafusa 1989).
Deadenylation and degradation proceeds in distinct phases

Our data suggest that there are distinct phases in the process of decay with regard to the poly(A) tail. First, immediately after transcriptional repression, we observed that for each mRNA there was a brief period before the maximum rate of deadenylation was achieved. This phenomenon is most easily seen with the MFA2 and GAL10 mRNAs (Figs. 2 and 4). During this period, the poly $(\mathrm{A})$ tails shortened relatively slowly compared with later times (see Table 1). We interpret these data to suggest that the initial shortening of the poly(A) tail, which we term initial deadenylation, may be distinctly different from subsequent events (see Fig. 9). Our data are most consistent with the transition to the second phase of deadenylation occurring at a specific time, as a result of some undefined event, rather than this transition requiring that the poly $(\mathrm{A})$ tails be shortened to a discrete length. This event may be nuclear-cytoplasmic transport, exchange of nuclear mRNP proteins for cytoplasmic mRNP proteins, completion of the first round of translation, or an undefined cytoplasmic localization event.

During the second phase, which we refer to as poly $(\mathrm{A})$ shortening (see Fig. 9), the poly(A) tail was shortened to an oligo(A) form of on average 7-16 nucleotides, with some variation between different mRNAs (see Table 1). This phase of poly(A) shortening was quite different for the individual mRNAs, both in terms of the rate of the reaction, which varied approximately fivefold, and in the uniformity of the behavior of the population. For instance, PGK1 mRNA was uniformly deadenylated dur-

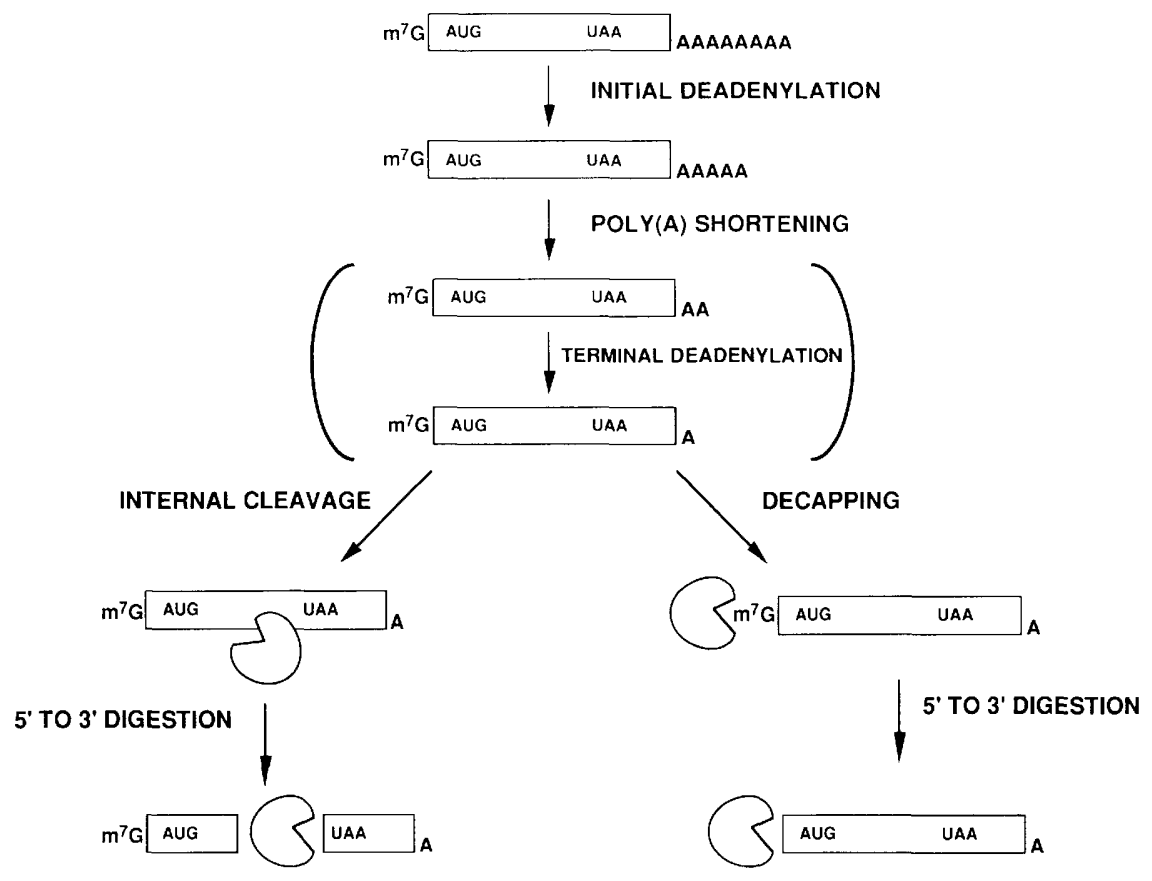

Figure 9. Model of a general, deadenylation-dependent mRNA decay pathway in yeast. Deadenylation occurs in distinct phases. During the first phase, initial deadenylation, the initial poly(A) tails are deadenylated slowly. During the second phase, poly(A) shortening, the poly(A) tails are shortened more rapidly to an oligo(A) length. Terminal deadenylation, referring to further shortening of the oligo(A) tail, is depicted in parentheses because our studies suggest that this phase in deadenylation is not obligatory for later steps in the pathway (see Discussion). The mRNA is subject to degradation after reaching the oligo(A) form. Deadenylation to an oligo(A) tail could stimulate degradation by affecting the efficiency of translational initiation, the mRNP structure, or the localization of the mRNA. These changes could promote internal endonucleolytic cleavage or the removal of the $5^{\prime}$ cap allowing the subsequent degradation of the mRNA by $5^{\prime}$ exonucleases. Different rates of the steps in this pathway between mRNAs results in the differential stability of mRNAs. 
ing this phase, whereas the other mRNAs had a more heterogeneous distribution of poly $(\mathrm{A})$ tail lengths. The basis for this heterogeneous behavior is not known but could be attributable to either different enzymatic activities functioning on particular mRNAs or to substratespecific differences in the functioning of a single poly(A) nuclease (Lowell et al. 1992). It should be noted that the observed rate of poly(A) shortening for a given mRNA may reflect a balance between deadenylation and cytoplasmic readenylation.

The deadenylation rate appeared to slow down once an oligo(A) tail was reached, representing a second transition in the deadenylation process. Any further deadenylation is referred to as terminal deadenylation (Fig. 9; Muhlrad and Parker 1992). Oligo(A) tails may be poor substrates for further deadenylation because they would be unable to bind efficiently to $\mathrm{PAB}$, the poly $(\underline{\mathrm{A}})$ binding protein, which prefers a binding site of 12 adenylate residues (Sachs et al. 1987) and has been proposed to activate a poly(A) nuclease (PAN1) (Lowell et al. 1992; Sachs and Deardorff 1992).

The final step in this pathway was the degradation of the oligo(A) species of the mRNA. Because individual mRNAs show differences in the rate of this step, it will be important to identify the rate determining event in the decay of the oligo(A) species. One possibility is that terminal deadenylation reduces the poly $(\mathrm{A})$ tail to below a critical length, followed extremely rapidly by subsequent events. Two observations are inconsistent with this model. First, the decay fragments that we observed for both PGK1 and MFA2 mRNAs had oligo(A) tails of approximately the same length as the oligo(A) tails on the full-length transcripts at the same time point (Fig. 8), clearly indicating that subsequent decay events do not require the complete removal of the poly(A) tail. In addition, although the chimeric PGK1-MFA2 mRNA undergoes rapid poly $(\mathrm{A})$ shortening and terminal deadenylation to a transcript with no, or very few, adenylate residues (Fig. 5), this deadenylated species still decayed at a rate similar to the oligo(A) form of $P G K 1$ mRNA. These observations suggest that events other than terminal deadenylation are rate determining for the degradation of the oligo(A) species. Therefore, the differences in the rate of decay of the oligo(A) species between mRNAs would be the result of transcripts having inherently different rates for this next, as yet undefined, step.

\section{Role of deadenylation in stimulating decay}

What is the role of deadenylation in stimulating decay? It has been proposed that deadenylation exposes the $3^{\prime}$ end of transcripts to nonspecific exonucleases (Brawerman 1987; Higgins 1991; Peltz et al. 1991; Shyu et al. 1991). The detecton of degradation intermediates that accumulate after deadenylation and contain only the $3^{\prime}$ portion of PGK1 and MFA2 mRNAs, however, is inconsistent with such a model. These data indicate instead that following deadenylation, the body of the transcript is degraded, at least partially, in a $5^{\prime} \rightarrow 3^{\prime}$ direction. Formally, the fragments that we detect could also be ex- plained by the presence of a poly $(G)$-specific endonuclease. Several observations suggest that this possibility is highly unlikely. First, the decay rate of the mRNAs was not altered by insertion of the poly(G). Second, consistent with the decay of the wild-type transcripts, the fragments appear only after deadenylation has occurred. Third, insertion of a different sequence, one that forms a stem-loop, into the 3' UTR of MFA2 mRNA gave similar results to the poly $(G)$ insertion (data not shown) consistent with the accumulation of these fragments being a result of secondary structures blocking exonuclease digestion. Given these observations, we conclude that the poly $(\mathrm{G})$ or the stem-loop act simply to block $5^{\prime} \rightarrow 3^{\prime}$ exonuclease digestion of the transcripts allowing the detection of normal events in the degradation of these mRNAs. As shown in Figure 9, two mechanisms by which these degradation intermediates could arise are by internal cleavage, which has been suggested to occur on the PGK1 mRNA (Vreken and Raué 1992), or by removal of the 5' cap structure by cleavage of the pyrophosphate bond between the cap and the mRNA. In each case, these events would be followed by $5^{\prime} \rightarrow 3^{\prime}$ exonucleolytic digestion.

Why would deadenylation stimulate cleavage events elsewhere on the mRNA? There are two general explanations for this phenomenon. One possibility is that cleavage is an indirect result of deadenylation. For instance, poly(A) tail shortening could alter subcellular localization of an mRNA (Lenk et al. 1977; Taneja et al. 1992) or decrease its rate of translational initiation (Doel and Carey 1976; Galili et al. 1988; Sachs and Davis 1989); these changes could then leave the mRNA susceptible to cleavage. Alternatively, deadenylation may have a more direct effect by disrupting interactions between the $3^{\prime}$ end of mRNAs and other sites on the RNA molecule that are dependent on the poly(A) tail (e.g., Bergmann and Brawerman 1977; Galili et al. 1988). Loss of such interactions could lead to alterations in mRNA or messenger ribonucleoprotein (mRNP) structure, thereby exposing sites within or at the $5^{\prime}$ end of the mRNA to cleavage.

\section{Implications for control of differential mRNA decay rates}

Our results suggest that at least some stable and unstable mRNAs are degraded by a pathway that shares the same basic mechanism. Therefore, the differences in the rates of the steps in this pathway are the basis for the observed half-lives of individual mRNAs. Two steps, poly(A) shortening and the decay of the oligo(A) species, appear to be the primary basis for the differences in mRNA decay rates because the rate of these steps varied the most between transcripts. For example, the extremely stable $P G K 1$ mRNA has a slow rate for both of these steps. In contrast, the unstable MFA2 mRNA completes both of these steps extremely rapidly. In this view, the intermediate decay rate of the GAL10 mRNA can be explained by this transcript being rapidly deadenylated followed by slow degradation of its oligoadenylated spe- 
cies. Another implication of our results is that the step(s) that contribute the most to the observed overall decay rate can vary between different mRNAs.

The observation that both stable and unstable mRNAs were degraded by a common mechanism implies that mRNAs contain sequences that can affect the rate of poly(A) shortening and the decay of the oligo(A) species, either by recruiting different nucleases or by modulating the activity of a small set of nucleases. Several examples of sequences that stimulate poly $(\mathrm{A})$ shortening rate have been identified, including the AU-rich element and coding region instability element of c-fos mRNA (e.g., Shyu et al. 1991) and sequences within the 3' UTR of MFA2 mRNA (Muhlrad and Parker 1992). Moreover, our results suggest that individual steps can be controlled by different mRNA features. For example, although the PGK1$M F A 2$ chimeric transcript underwent rapid poly(A) shortening, similar to the $M F A 2$ transcript, the stability of the oligo(A) species was similar to that of the PGK1 transcript. Therefore, although the 3' UTR of MFA2 mRNA was sufficient to promote rapid deadenylation, features within the PGK1 sequences appear to dictate the rate of decay of the oligo(A) species.

\section{Gene products involved in deadenylation and degradation}

The complexity of the deadenylation and degradation process suggests that several factors will be involved. Proteins identified in yeast that may play a role in deadenylation include PAB (Sachs and Davis 1989), a PABdependent poly $(A)$ nuclease (PAN1) (Sachs and Deardorff 1992), and the products of the RNA14 and RNA15 genes (Bloch et al. 1978; Minvielle-Sebastia et al. 1991). Strains deficient in $\mathrm{PAB}$ accumulate long poly(A) tails. In contrast, mutations in the RNA14 and RNA15 genes lead to decreased levels of total poly(A) and an increase in the rate of decay of the stable $A C T 1$ mRNA, suggesting that these gene products normally function to inhibit the rate of deadenylation. Activities that may be involved in the events following deadenylation have also been identified. Two $5^{\prime} \rightarrow 3^{\prime}$ exonucleases, which account for the majority of exonuclease activity in crude yeast extracts, have been cloned (Larimer and Stevens 1990; Kenna et al. 1993). Interestingly, one of these nucleases biochemically copurifies with an activity that preferentially decaps long mRNA substrates (Stevens 1988). Future experiments using the protocol described in this paper should illuminate the roles these gene products play in deadenylation and decay.

\section{Deadenylation-dependent decay in other eukaryotes}

Several observations suggest that the pathway of mRNA decay we have described in yeast also occurs in other eukaryotic cells. At least a small subset of mammalian mRNAs require deadenylation before decay (Brewer and Ross 1988; Wilson and Triesman 1988; Swartwout and Kinniburgh 1989; Laird-Offringa et al. 1990; Shyu et al. 1991). Moreover, in a number of studies, changes in the size distribution of poly(A) tails are correlated with alterations in mRNA decay rates (for review, see Bernstein and Ross 1989; Peltz et al. 1991). In addition, for several mRNAs, a portion $(\sim 25$ or more adenylate residues) of the poly(A) tail persists after shortening of the majority of the poly $(\mathrm{A})$ tail has occurred, indicating that there are at least two phases of deadenylation in eukaryotes other than yeast (Mercer and Wake 1985; Restifo and Guild 1986; Kleene 1989; Shyu et al. 1991). Given the fundamental similarity of other physiological processes between yeast and more complex eukaryotes (e.g., Lee and Nurse 1987; Shuster and Guthrie 1990), we speculate that additional steps in this yeast decay pathway may occur in an analogous manner in complex eukaryotes.

\section{Materials and methods}

\section{Yeast strains}

The yeast strains used in this study were derived from crossing JRY1606 (MAT $\alpha$, ade2, Iys2, ura3-52; provided by J. Rine, University of California, Berkeley) with N218 (MATa, rpb1-1, ura352 , his3, leu2; provided by M. Nonet and R. Young, The Whitehead Institute, Cambridge, MA). The strains used for the experiments were yRP582 (MATa, rpb1-1, ura3-52, leu2), yRP583 (MAT $\alpha$, rpb1-1, ura3-52, ade2, lys2, leu2), and yRP673. yRP673 was obtained by converting the mating type of yRP582 with the HO endonuclease (Herskowitz and Jensen 1991). Strains were transformed with plasmids by use of a modification of the lithium acetate method as described in Schiestle and Gietz (1989). Synthetic media lacking uracil was used to select and maintain plasmids.

\section{Construction of plasmids}

The construction of $\mathrm{pRP} 22$, the yeast vector used in these experiments, and pRP170, which encodes PGK1 marked with an oligonucleotide in its $3^{\prime}$ UTR and expressed under galactose transcriptional regulation, is described in Heaton et al. (1992).

The plasmid that allows MFA2 mRNA to be expressed under galactose regulation was made by BAL 31 digestion from the SphI site of pSM29 (Michealis and Herskowitz 1989) which contains the $M F A 2$ gene and flanking sequences, and subcloning of fragments containing the MFA2 gene into the SmaI and HindIII sites of pRP22. The resulting plasmids were screened for galactose induction and glucose repression of $M F A 2$ gene expression. The $5^{\prime}$ end of MFA2 sequences in the selected plasmid pRP410 was determined by sequencing to be upstream of the transcriptional start site, 238 bases $5^{\prime}$ of the translational start codon.

The STE3 gene was fused with the GAL1 UAS as follows. The plasmid CY809 containing a $2.7-\mathrm{kb}$ HindIII fragment encompassing the STE3 gene in YCp50 (Sprague et al. 1983; provided by G. Sprague) was cut with StuI, which cleaves 604 bases upstream of the translational start codon, digested with BAL 31, filled in with Klenow, cleaved with $S p h I$, and inserted into the SmaI and SphI sites of pRP22. The resulting plasmids were screened for galactose-regulated STE3 mRNA expression. The $5^{\prime}$ end of STE3 sequences in the selected plasmid pRP465 was determined by sequencing to be upstream of the transcriptional start site, 262 bases $5^{\prime}$ of the translational start codon.

The chimeric PGK1-MFA2 gene was constructed by replacement of the ClaI-HindIII fragment of pRP170 containing the 3' UTR of PGK1 with an $\sim 670$-bp BamHI-HindIII fragment from 
MFA2 containing sequences just $5^{\prime}$ of the translational stop codon and extending beyond the 3' UTR yielding pRP430.

The insertion of poly $(G)$ into $P G K 1$ yielded a plasmid (pRP469) identical to pRP170, except the oligonucleotide marking PGK1 in the ClaI site in the $3^{\prime}$ UTR was replaced with the sequence CGAAGGAATTTGGGGGGGGGGGGGGGGGGAATTCCT.

The poly $(G)$ insertion into MFA2 yielded a plasmid (pRP485) identical to pRP410 except that it had the sequence GATCTAGGAATTTGGGGGGGGGGGGGGGGGGAATTCCT inserted into a previously constructed BgIII site, B178 (Muhlrad and Parker 1992).

Procedure to produce and follow newly synthesized pools of specific mRNAs

Synthetic medium $(200 \mathrm{ml})$ containing $2 \%$ raffinose was inoculated with cells pregrown on YEP $2 \%$ raffinose plates. Cultures were grown at $24^{\circ} \mathrm{C}$ until they reached mid-log phase $\left\{\mathrm{OD}_{600}\right.$ $\sim 0.3$, harvested by spinning for $2 \mathrm{~min}$ at top speed in a tabletop centrifuge, resuspended in $10 \mathrm{ml}$ of media containing $2 \%$ raffinose at $24^{\circ} \mathrm{C}$ and shaken at $24^{\circ} \mathrm{C}$ for $10 \mathrm{~min}$. Transcription was induced by adding $40 \%$ galactose to a final concentration of $2 \%$. An aliquot was removed immediately for an uninduced control. A second aliquot was removed after $10-15 \mathrm{~min}$. The culture was then transferred immediately to a preheated flask at $36^{\circ} \mathrm{C}$ and an equal volume of medium containing $4 \%$ glucose at $58^{\circ} \mathrm{C}$ was added (final concentration of glucose was $2 \%$ ). Use of media at $58^{\circ} \mathrm{C}$ ensures the rapid equilibration of the temperature of the culture to $36^{\circ} \mathrm{C}$, the restrictive temperature for $r p b 1-1$. Aliquots were then removed at various times and harvested by centrifugation for $30 \mathrm{sec}$ in a microcentrifuge, followed by aspiration of the supernatant and quick freezing of the cell pellet on dry ice.

Two sets of observations indicate that the results obtained using this procedure are not an artifact of the method. First, similar results were seen when transcription was inhibited only by glucose addition, thus avoiding any heat shock and the effects of a general repression of RNA polymerase II and when transcription was repressed only by temperature shift, thus avoiding any possible artifacts induced by carbon source changes. In addition, the distribution of poly(A) tail lengths in steady-state populations of the transcripts examined were consistent with what would be predicted from the various rates measured in our induction experiments. For example, oligoadenylated species were abundant in steady-state populations of both PGK1 and GAL10 transcripts but not in steady-state populations of either MFA2 or STE3 transcripts.

\section{Analysis of poly $(A)$ tail length and $m R N A$ decay rate}

Total yeast RNA was isolated from the cell pellets as described in Caponigro et al. (1993). mRNA sizes were estimated using $5 '$-end-labeled HpaII pUC18 DNA or HinfI $\Phi$ X174 DNA as size markers. The lengths of poly(A) tails on MFA2 mRNA were determined by direct comparison of the size of untreated MFA2 mRNA with its size after removal of the poly(A) tail by hybridization with oligo(dT) and digestion with RNase $\mathrm{H}$. To resolve the contribution of the poly|A) tail to the length of PGK1, GAL1O, and STE3 mRNAs, these mRNAs were first cleaved with oligonucleotides specific for the $3^{\prime}$ end of the respective RNA (as described in the figure legends) and RNase H. Ten micrograms of total RNA was hybridized with $200-300 \mathrm{ng}$ of mRNA-specific oligonucleotide plus or minus $300 \mathrm{ng}$ of oligo/dT) (Pharmacia) and incubated with 0.025 units/ $\mu$ l of RNase $\mathrm{H}$ in $20 \mathrm{~mm}$ Tris-Cl (pH 7.5), $10 \mathrm{~mm} \mathrm{MgCl}, 0.5 \mathrm{~mm}$ EDTA, 50 $\mathrm{mM} \mathrm{NaCl}, 1 \mathrm{~mm}$ DTT, and $30 \mu \mathrm{g} / \mathrm{ml}$ of BSA for $1 \mathrm{hr}$ at $30^{\circ} \mathrm{C}$.
RNA was resolved on $6 \%$ polyacrylamide $/ 7.7 \mathrm{M}$ urea gels, transferred using an electroblotter (Hoeffer) to Zeta-probe membrane and hybridized by use of standard methods. Hybridization to blots was quantitated using a Betascope (Betagen, Waltham, MA). The amount of specific mRNA in each lane was normalized using the yeast transcript scR1, the homolog to mammalian 7S RNA that is transcribed by RNA polymerase III (Felici et al. 1989).

\section{Acknowledgments}

We thank Jasper Rine, Michael Nonet, and Richard Young for yeast strains used in this study; Denise Muhlrad for her technical assistance; and Ellen Baker, John Little, Carol Dieckmann, Robin Staples, and the members of R.P.'s laboratory for helpful comments on the manuscript. This work was supported by grants to R.P. from the Chicago Community Trust and and National Institutes of Health (GM45443) and a fellowship (DRG-1141) from the Damon Runyon-Walter Winchell Cancer Research Fund to C.D.

The publication costs of this article were defrayed in part by payment of page charges. This article must therefore be hereby marked "advertisement" in accordance with 18 USC section 1734 solely to indicate this fact.

\section{References}

Bergmann, I.E. and G. Brawerman. 1977. Control of the breakdown of the polyadenylate sequence in mammalian polyribosomes: Role of poly(adenylic acid)-protein interactions. Biochemistry 16: 259-264.

Bernstein, P. and J. Ross. 1989. Poly(A), poly(A) binding protein and the regulation of mRNA stability. Trends Biochem. Sci. 14: 373-377.

Binder, R., S.-P.L. Hwang, R. Ratnasabapathy, and D. L. Williams. 1989. Degradation of apolipoprotein II mRNA occurs via endonucleolytic cleavage at $5^{\prime}$-AAU- $3^{\prime} / 5^{\prime}$-UAA-3' elements in single-stranded loop domains of the $3^{\prime}$ non-coding region. J. Biol. Chem. 264: 16910-16918.

Bloch, J.C., F. Perrin, and F. Lacroute. 1978. Yeast temperaturesensitive mutants specifically impaired in processing of poly(A)-containing RNAs. Mol. Gen. Genet. 165: 123-127.

Brawerman, G. 1987. Determinants of messenger RNA stability. Cell 48: 5-6.

Brewer, G. and J. Ross. 1988. Poly(A) shortening and degradation of the $3^{\prime} \mathrm{A}+\mathrm{U}$-rich sequences of human c-myc mRNA in a cell free system. Mol. Cell. Biol. 8: 1697-1708.

Caponigro, G., D. Muhlrad, and R. Parker. 1993. A small segment of the MAT $\alpha 1$ transcript promotes mRNA decay in yeast: a stimulatory role for rare codons. Mol. Cell. Biol. (in press).

Cleveland, D.W. and T.J. Yen. 1989. Multiple determinants of eukaryotic mRNA stability. New Biol. 1: 121-126.

Citron, B.A. and J.E. Donelson. 1984. Sequence of the Saccharomyces GAL region and its transcription in vivo. J. Bacteriol. 158: 269-278.

Doel, M.T. and N.H. Carey. 1976. The translational capacity of deadenylated ovalbumin messenger RNA. Cell 8: 51-58.

Felici, F., G. Cesareni, and J.M.X. Hughes. 1989. The most abundant small cytoplasmic RNA of Saccharomyces cerevisiae has an important function required for normal cell growth. Mol. Cell. Biol. 9: 3260-3268.

Galili, G., E.E. Kawata, L.D. Smith, and B.A. Larkins. 1988. Role of the 3 -poly $(A)$ sequence in translational regulation of mRNAs in Xenopus laevis oocytes. J. Biol. Chem. 
263: $5764-5770$.

Heaton, B., C. Decker, D. Muhlrad, J. Donahue, A. Jacobson, and R. Parker. 1992. Analysis of chimeric mRNAs identifies multiple regions within the STE3 mRNA which promote rapid mRNA decay. Nucleic Acids Res. 20: 5365-5373.

Herrick, D., R. Parker, and A. Jacobson. 1990. Identification and comparison of stable and unstable mRNAs in Saccharomyces cerevisiae. Mol. Cell. Biol. 10: 2269-2284.

Herskowitz, I. and R.E. Jensen. 1991. Putting the HO gene to work: Practical uses for mating-type switching. Guide to yeast genetics and molecular biology. Methods Enzymol. 194: $132-146$.

Higgins, C.F. 1991. Stability and degradation of mRNA. Curr. Opin. Cell Biol. 3: 1013-1018.

Johnston, M. 1987. Model fungal gene regulatory mechanism: The GAL genes of Saccharomyces cerevisiae. Biol. Rev. 51: 458-476.

Kenna, M., A. Stevens, M. McCammon, and M.G. Douglas. 1993. An essential yeast gene with homology to the exonuclease-encoding XRN1/KEMl gene also encodes a protein with exoribonuclease activity. Mol. Cell. Biol. 13: 341-350.

Kleene, K.C. 1989. Poly(A) shortening accompanies the activation of translation of five mRNAs during spermiogenesis in the mouse. Development 106: 367-373.

Krowczynska, A., R. Yenofsky, and G. Brawerman. 1985. Regulation of messenger RNA stability in mouse erythroleukemia cells. I. Mol. Biol. 181: 231-239.

Laird-Offringa, I.A., C.L. de Wit, P. Elfferich, and A.J. van der Eb. 1990. Poly(A) tail shortening is the translation-dependent step in c-myc mRNA degradation. Mol. Cell. Biol. 10: 61326140 .

Larimer, F.W. and A. Stevens. 1990. Disruption of the gene XRN1, coding for a $5^{\prime}$ to $3^{\prime}$ exoribonuclease, restricts yeast cell growth. Gene 95: 85-90.

Lee, M.G. and P. Nurse. 1987. Complementation used to clone a human homologue of the fission yeast cell cycle control gene cdc2. Nature 327: 31-35.

Lenk, R., L. Ransom, Y. Kaufmann, and S. Penman. 1977. A cytoskeletal structure with associated polyribosomes obtained from HeLa cells. Cell 10: 67-78.

Lowell, J.E., D.Z. Rudner, and A.B. Sachs. 1992. 3'-UTR-dependent deadenylation by the yeast poly(A) nuclease. Genes \& Dev. 6: 2088-2099.

McLaren, R.S., S.F. Newbury, G.S.C. Dance, H.C. Causton, and C.F. Higgins. 1991. mRNA degradation by processive $3^{\prime}-5^{\prime}$ exoribonucleases in vitro and the implications for prokaryotic mRNA decay in vivo. $J$. Mol. Biol. 220: 81-95.

Mercer, J.B. and S.A. Wake. 1985. An analysis of the rate of metallothionein mRNA poly(A)-shortening using RNA blot hybridization. Nucleic Acids Res. 13: 7929-7943.

Michealis, S. and I. Herskowitz. 1989. The a-factor pheromone of Saccharomyces cerevisiae is essential for mating. Mol. Cell. Biol. 8: 1309-1318.

Minvielle-Sebastia, L., B. Winsor, N. Bonneaud, and F. Lacroute. 1991. Mutations in the yeast RNA14 and RNA15 genes result in an abnormal mRNA decay rate; sequence analysis reveals an RNA-binding domain in the RNA15 protein. Mol. Cell. Biol. 11: 3075-3087.

Muhlrad, D. and R. Parker. 1992. Mutations affecting stability and deadenylation of the yeast MFA2 transcript. Genes \& Dev. 6: 2100-2111.

Nonet, M., C. Scafe, J. Sexton, and R. Young. 1987. Eukaryotic RNA polymerase conditional mutant that rapidly ceases mRNA synthesis. Mol. Cell. Biol. 7: 1602-1611.

Parker, R., D. Muhlrad, J.O. Deshler, N. Taylor, and J.J. Rossi. 1992. Ribozymes: Principles and designs for their use as an- tisense and therapeutic agents. In Gene regulation: Biology of antisense RNA and DNA (ed. R.P. Erickson and J.G. Izant), pp. 55-70. Raven Press, New York.

Peltz, S.W., G. Brewer, P. Bernstein, P.A. Hart, and J. Ross. 1991. Regulation of mRNA turnover in eukaryotic cells. Crit. Rev. Euk. Gene Expression 1: 99-126.

Restifo, L.L. and G.M. Guild. 1986. Poly(A) tail shortening of coregulated transcripts in Drosophila. Dev. Biol. 115: 507510.

Sachs, A.B. and R.W. Davis. 1989. The poly(A) binding protein is required for poly(A) shortening and $60 \mathrm{~S}$ ribosomal subunitdependent translation initiation. Cell 58: 857-867.

Sachs, A.B. and J.A. Deardorff. 1992. Translation initiation requires a PAB-dependent poly(A) ribonuclease in yeast. Cell 70: $961-973$.

Sachs, A.B., R.W. Davis, and R.D. Kornberg. 1987. A single domain of yeast poly(A)-binding protein is necessary and sufficient for RNA binding and cell viability. Mol. Cell. Biol. 7: 3268-3276.

Scheiness, D. and J.E. Darnell. 1973. Polyadenylic acid segment in mRNA becomes shorter with age. Nature New Biol. 241: 265-268.

Schiestl, R.H. and R.D. Gietz. 1989. High efficiency transformation of intact yeast cells using single stranded nucleic acids as a carrier. Curr. Genet. 16: 339-346.

Shuster, E.O. and C. Guthrie. 1990. Human U2 snRNA can function in pre-mRNA splicing in yeast. Nature 345: 270273.

Shyu, A-B., J.G. Belasco, and M.E. Greenberg. 1991. Two distinct destabilizing elements in the c-fos message trigger deadenylation as a first step in rapid mRNA decay. Genes \& Dev. 5: 221-234.

Sprague, G.F., Jr., R. Jensen, and I. Herskowitz. 1983. Control of yeast cell type by the mating type locus: Positive regulation of the $\alpha$-specific STE3 gene by the MAT $\alpha 1$ product. Cell 32: 409-415.

Stevens, A. 1988. mRNA-decapping enzyme from Saccharomyces cerevisiae: Purification and unique specificity for long RNA chains. Mol. Cell. Biol. 8: 2005-2010.

Stoeckle, M.Y. and H. Hanafusa. 1989. Processing of 9E3 mRNA and regulation of its stability in normal and rous sarcoma virus-transformed cells. Mol. Cell. Biol. 9: 4738-4745.

Swartwout, S.G. and A.J. Kinniburgh. 1989. c-myc mRNA degradation in growing and differentiating cells: Possible alternative pathways. Mol. Cell. Biol. 9: 288-295.

Taneja, K.L., L.M. Lifshitz, F.S. Fay, and R.H. Singer. 1992. Poly(A) RNA codistribution with microfilaments: Evaluation by in situ hybridization and quantitative digital imaging microscopy. J. Cell Biol. 119: 1245-1260.

Vreken, P. and H.A. Raué. 1992. The rate-limiting step in yeast PGK 1 mRNA degradation is an endonucleolytic cleavage in the 3 '-terminal part of the coding region. Mol. Cell. Biol. 12: 2986-2996.

Wickens, M. 1990. How the messenger got its tail: Addition of poly(A) in the nucleus. Trends Biochem. Sci. 15: 277-281.

Williamson, J.R., M.K. Ragharaman, and T.R. Cech. 1989. Monovalent cation-induced structure of telomeric DNA: The G-quartet model. Cell 59: 871-880.

Wilson, T. and R. Triesman. 1988. Removal of poly(A) and consequent degradation of the c-fos mRNA facilitated by $3^{\prime}$ AUrich sequences. Nature 336: 396-399.

Zimmerman, S.B., G.H. Cohen, and D.R. Davies. 1975. X-ray fiber diffraction and model-building study of polyguanylic acid and polyinosinic acid. J. Mol. Biol. 92: 181-192. 


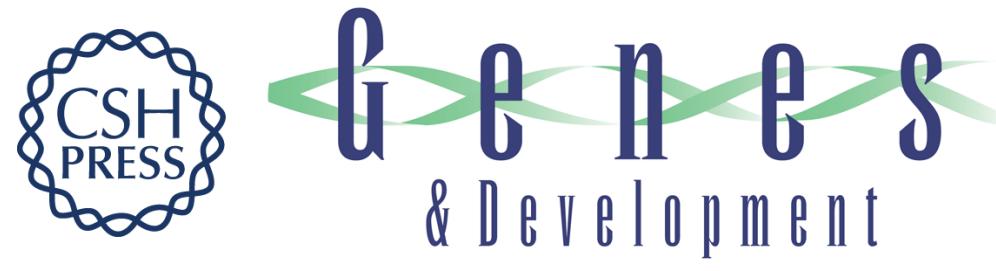

\section{A turnover pathway for both stable and unstable mRNAs in yeast: evidence for a requirement for deadenylation.}

C J Decker and R Parker

Genes Dev. 1993, 7:

Access the most recent version at doi:10.1101/gad.7.8.1632

References This article cites 49 articles, 20 of which can be accessed free at:

http://genesdev.cshlp.org/content/7/8/1632.full.html\#ref-list-1

License

Email Alerting

Service

Receive free email alerts when new articles cite this article - sign up in the box at the top right corner of the article or click here.

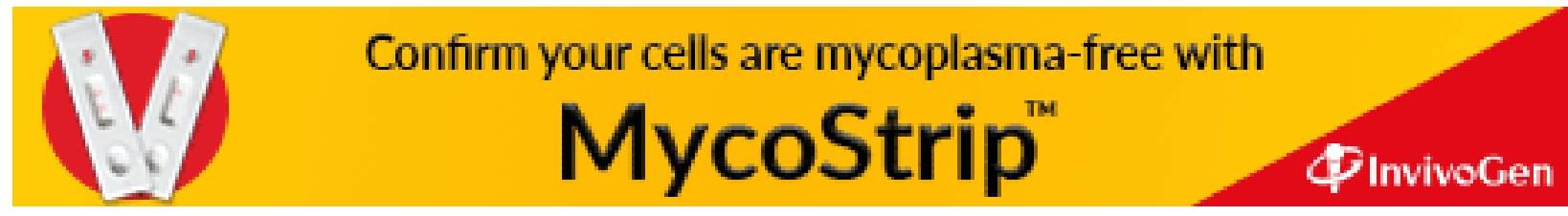

